\title{
Donepezil hydrochloride: a double-blind study in autistic children
}

\author{
Michael G. Chez, Thomas M. Buchanan, Marina Becker, \\ Jami Kessler, Mary C. Aimonovitch, Susan R. Mrazek
}

Autism and Epilepsy Services of Illinois, S.C., S.C.; Lake Bluff, Illinois, U.S.A.

\begin{abstract}
Recent studies in autistic brain samples have shown diminished acetylcholine and nicotinic receptor activity. We hypothesized that acetylcholinergic enhancement may pharmacologically improve some autistic characteristics. Donepezil hydrochloride, an acetylcholinesterase inhibitor, was studied previously in two open label studies which showed improvement in the expressive and receptive speech and aberrant behaviors of autistic children. We therefore undertook a double-blind placebo controlled study to confirm these findings. Forty-three patients (35 males, 8 females, average age 6.8 yrs., range 2.1-10.3 yrs), with diagnoses of Autistic Spectrum Disorders enrolled in a randomized six-week, doubleblind, placebo-controlled trial of donepezil hydrochloride, with an additional six weeks of open-label treatment. Change was evaluated by the Childhood Autistic Rating Scale, Gardner's Expressive One-Word Picture Vocabulary Test, Revised, and Gardner's Receptive OneWord Picture Vocabulary Test. Testing was administered at baseline, six-week, and twelveweek follow-up. Expressive and receptive speech gains, as well as decreases in severity of overall autistic behavior, were documented after 6-weeks for the treatment group. These improvements were statistically significant when compared to placebo, and were clinically meaningful as assessed over time. Donepezil hydrochloride
\end{abstract}

Correspondence: Michael G. Chez, M.D.

Director, Autism and Epilepsy Services of

Illinois, S.C. 40 East Scranton

P.O. Box 280 Lake Bluff, Illinois 60044, U.S.A.

Tel: (847) 735-0300, fax: (847) 735-0400.

E-mail: mchezmd@sbcglobal.net

Received: May 21, 2003.

Revised: November 13, 2003.

Accepted: November 14, 2003. appears to improve expressive and receptive language as well as overall autistic features, consistent with the hypothesis of acetylcholinergic enhancement. (J Pediatr Neurol 2003; 1(2): 8388).

Key words: autism, autistic spectrum disorder, donepezil hydrochloride.

\section{Introduction}

The search for a single anatomical or neurochemical defect in autism has been unsuccessful to date. However, recent research by Elaine Perry and her colleagues (1) examined basal forebrain and cerebral cortex of adult autistic brains, and described frontal nicotinic receptor type $a 4 b 2$ as significantly reduced in Autistic Spectrum Disorders (ASD) in the frontal lobe region versus controls. Parietal muscarinic receptors were also reduced compared to normal controls (1). These findings support the current study hypothesis that pharmacotherapy may ameliorate acetylcholinergic abnormalities that may exist in autistic children. They indicate success in ASD treatment for a drug that could restore normal functioning to acetylcholinergic receptors by increasing the concentration of available acetylcholine.

Pharmacological therapy of frontal lobe dementia and Alzheimer's disease has been targeted at acetylcholine-producing neurons. Donepezil hydrochloride (DH) is believed to be a specific and potent acetylcholinesterase (AChE) inhibitor (2). $\mathrm{DH}$ has also been used to treat pediatric patients with Tourette's syndrome and attention deficit hyperactivity disorders (ADHD) $(3,4)$ on the theory that DH increases acetylcholine levels and modulates nicotinic receptors, which lower dopamine levels and improve tics. There has also been a report of successful clinical benefit for patients with Down's syndrome describing increased speech output and enhanced cognitive function (5).

We therefore proposed that given the underlying evidence of frontal apraxia and expressive speech 
deficits, DH could allow for similar gains in a pediatric ASD sample. Our recent open-label trial with $\mathrm{DH}$ demonstrated expressive language improvements from baseline to 12-week follow-up as determined by Gardner's Expressive One-Word Picture Vocabulary Test, Revised (EOWPVT-R); improvements attained statistical significance $(\mathrm{P}=$ 0.032). Subjects gained an average of eight months in expressive speech according to language age equivalencies (6). Another recent retrospective open-label trial with $\mathrm{DH}$ showed improvements in scores on the Aberrant Behavior Checklist and the Clinical Global Impression Scale in four of eight autistic children tested over a period of two months (7). The present study evaluated changes in clinical features of autistic spectrum disorder using $\mathrm{DH}$ in a six week, double-blind parallel group design with an open label extension.

\section{Materials and Methods}

\section{Participants}

Forty-three patients (35 males, 8 females, mean $\pm \mathrm{SD}, 6.8 \pm 1.9$ yrs., range 2.1-10.3 yrs) enrolled in a six week double-blind parallel group design study with an open label extension. Patients were randomized to receive either (1) twelve weeks of $\mathrm{DH}$ or (2) six weeks of placebo followed by six weeks of DH. Children were asked to participate in consecutive order of entry into the office for regularly scheduled office visit. Inclusion criteria were: males or females, ages 2-10 with prior diagnoses of Autistic Disorder $(n=13)$ or Pervasive Developmental Disorder-Not Otherwise Specified $(n=27)$ or Landau-Kleffner Syndrome $(n=3)$ as outlined by clinical criteria in the Diagnostic and Statistical Manual (DSM-IV) (8). Diagnosis was confirmed by a pediatric neurologist after completing a comprehensive neurological evaluation and also by a clinical interview with a clinical psychologist. No child was excluded based upon intelligence estimate or developmental language level. Children were not eligible for participation if they had a concomitant neurological syndrome or disease in which neurological compromise is a feature (e.g., neurofibromatosis).

We included patients with abnormal electroencephalogram (EEG) as they represent an important section of the ASD population (9). Twenty-eight participants (72\%) had been previously diagnosed with nocturnal epileptiform EEG abnormalities, while 11 children (28\%) had normal 24-hour EEG patterns. Four had never had an EEG. Patients were maintained on the medications that they had initiated prior to study start: anticonvulsants $(n=32$, divalproex sodium, valproic acid or lamotrigine), the leading treatment for ASD epileptiform abnormalities $(9,10)$. Other patients were maintained on corticosteroids ( $n=6$, pulsedose prednisone or prednisolone), central nervous system (CNS) stimulants $(n=8$, dextroamphetamine / amphetamine or methylphenidate), antidepressants $(\mathrm{n}=7$, fluoxetine hydrochloride or paroxetine), antipsychotics $(\mathrm{n}=4, \quad$ risperidone $)$, or alpha adrenergic blocking agents $(n=9$, clonidine).

Participation in the study was voluntary and no compensation for participation was given. The study coordinators obtained written informed consent approved by the Lake Forest Hospital Institutional Review Board from one parent at the baseline visit.

\section{Procedures}

The key to the blinding code was kept with a staff member not involved with patient care or other aspects of the research study. At the baseline visit, group assignments were randomized by this unblinded staff member who assigned patients to the two groups as they enrolled in alternating fashion. This staff member informed the pharmacist whether a patient was to take placebo or $\mathrm{DH}$ and the pills were put in a bottle with only the code number and patient name identifying its contents. All other members of the medical staff, including the physician, were blind to this code.

Group one receiving 2.5 milligrams of $\mathrm{DH}$ and group two receiving placebo. The standard starting donepezil dosage recommendation of 5 milligrams was lowered in accordance with pediatric protocols to arrive at a quantity of 2.5 milligrams. Our prior experience had showed that this dose was well tolerated in a prior open label study (6). The duration of the double-blind, parallel trial was six weeks, at which time all subjects entered open-label treatment for an additional six weeks.

\section{Medication}

Placebo and DH were identical in appearance in capsule (sprinkle) form to allow for easy oral administration. The dose was lowered to 1.25 milligrams or discontinued throughout the 12 week trial if adverse events persisted for more than one week (e.g., irritability, hyperactivity, or gastrointestinal problems). The maximum daily dose was 2.5 milligrams. The medication was adjusted by halving the dose $(1.25 \mathrm{mg})$ if parents noted their children to have tolerable yet persistent gastrointestinal distress, hyperactivity, or irritability. Medication was discontinued if gastrointestinal intolerability or changes in behavior were considered to be an adverse event (i.e., persisted for more than one day). Blinding of the physician was maintained during adjustments in the dose of either placebo or DH. The physician in the study made the adjustments by changing the dose to the patient, without knowing whether the pill was donepezil or placebo. Medication was administered 
Table 1. Combination of group I with group II subjects treated with DH for six weeks

\begin{tabular}{llll}
\hline \hline & $\begin{array}{l}\text { Baseline } \\
\text { (mean } \pm \text { SD) }\end{array}$ & $\begin{array}{l}\text { 6-week } \\
\text { (mean } \pm \text { SD) }\end{array}$ & P value \\
\hline CARS & $34.7 \pm 7.4$ & $32.2 \pm 8.5$ & 0.004 \\
Exp. speech age (year) & $39.3 \pm 27.9$ & $43.6 \pm 31.8$ & 0.044 \\
Rec. speech age (year) & $43.7 \pm 22.2$ & $53.2 \pm 25.8$ & 0.002 \\
& & & \\
\hline \hline
\end{tabular}

at dinnertime, unless restlessness occurred in sleep, in which case administration was changed to an earlier time in the day. Dinner was selected as the preferred administration time in an attempt to alleviate gastrointestinal side effects observed in the dementia population.

Patient progress and side effects were monitored at the scheduled appointments by study coordinators, nursing staff, and the principal investigator. Weekly telephone contact with parents was documented in telephone logs. The most notable side effects reported in parental diaries and phone conversations were irritability, troubled sleep patterns, and gastrointestinal symptoms of nausea, decreased appetite, or diarrhea.

Parents were not informed whether their child was on placebo or $\mathrm{DH}$ until the conclusion of the study. All members of the medical staff, including nurses and the physician were blinded to the status of the patients on either placebo or drug during the six-week double-blind phase. The key to the blinding code was kept by an office staff member not involved with patient contact. Patient progress and side effects were monitored at the scheduled appointments by study coordinators, nursing staff, and the primary investigator. Patients that were removed from the study dues to side effects had their blinding code broken. Gardner's Receptive One-Word Picture Vocabulary Test (ROWPVT), the EOWPVT-R, and the Childhood Autism Rating Scale (CARS) were administered at baseline, 6week, and 12-week follow-up visits by the same study coordinator.

\section{Outcome Measures}

The Receptive One-Word Picture Vocabulary Test 11 examines a child's understanding of language and is an effective tool for assessing receptive language in children with expressive language difficulties. The test was developed for children ages two through twelve. Participants are shown a group of four pictures and asked to point to the picture corresponding to the word stated by the clinician. The test is completed when the child makes six errors of eight consecutive items.

The Expressive One-Word Picture Vocabulary
Test - Revised can be used as a tool to determine auditory-visual-verbal association ability as well as expressive language functioning in young children ages two through twelve. The test was developed to address general concrete concepts, grouping, abstract concepts, and descriptive concepts. Participants are shown a black-and-white picture and asked to identify it verbally. The test is completed when the child makes six consecutive errors.

Patients whose expressive or receptive language skills were not testible received a score of 0 for that test point.

The Childhood Autism Rating Scale was used to assess changes in autistic features (13). It was designed as a rating system for identification of behavioral symptoms and for classification purposes by replacing subjective clinical judgments with objective and quantifiable ratings based upon direct behavioral observation. We adapted the interview-based clinician assessment to a parental rating report that was completed together with the research coordinator at the time of the 6 and 12 -week clinic appointments. We asked that the same parent complete the CARS scale each time for the sake of consistency and homogeneity in assessment. The study coordinators asked the parent to form an impression of the child from the last six weeks when completing the CARS at each visit. The CARS is divided into 15 subcategories, each scored by increments of 0.5 points on a scale of one through four, which are cumulatively added resulting in a total score ranging from 15 to 60 . The rating scale for total scores is then divided into three classifications: non-autistic (15.0-30.0), mildly/moderately autistic (30.5-36.0), and severely autistic (36.5-60.0). A lower total score, therefore, designates a less severe rating.

Data were analyzed using the SPSS statistical package, version 10.0. Independent samples $t$-tests were conducted for the planned contrasts between group I and group II. Paired samples $t$-tests were conducted for analyses of change in CARS, EOWPVT-R, and ROWPVT scores within groups. A retrospective power analysis was performed for the pooled 6-week CARS, EOWPVT-R, and ROWPVT scores shown in Table 1. 
Table 2. Characteristics of study subjects

\begin{tabular}{lll}
\hline \hline & Group I $(\mathbf{n = 2 3 )}$ & Group II $(\mathbf{n}=\mathbf{2 0})$ \\
\hline Baseline & $(\mathrm{DH}, \mathrm{DH})$ & (Placebo, DH) \\
Mean age (yrs.) & 6.8 (range 2.1-9.9) & 6.9 (range 4.1-10.3) \\
Male/female & $19 / 4$ & $16 / 4$ \\
PDD/Autism/LKS & $14 / 8 / 1$ & $13 / 5 / 2$ \\
& & \\
Medications & 1 & 8 \\
Alpha adrenergic & 20 & 12 \\
Anticonvulsant & 4 & 4 \\
Antipsychotic & 4 & 2 \\
Steroids & 2 & 5 \\
Antidepressants & 5 & 3 \\
Stimulants & & \\
\hline \hline
\end{tabular}

Both groups were statistically equivalent at baseline for all variables.

PDD: Pervasive developmental disorder; LKS; Landau-Kleffner syndrome.

Table 3. Changes in behavior and language measures

\begin{tabular}{llll}
\hline \hline Group I (DH, DH) & $\begin{array}{l}\text { Baseline } \\
(\text { mean } \pm \text { SD) }\end{array}$ & $\begin{array}{l}\text { 6-week } \\
\text { (mean } \pm \text { SD) }\end{array}$ & $\begin{array}{l}\text { 12-week } \\
\text { (mean } \pm \text { SD) }\end{array}$ \\
\hline $\mathrm{n}$ & 23 & 17 & 17 \\
CARS & $34.7 \pm 7.7$ & $33.3 \pm 8.0$ & $30.8 \pm 7.9 * *$ \\
Exp. speech age (month) & $35.7 \pm 27.8$ & $43.3 \pm 27.2 *$ & $42.5 \pm 28.5 * * *$ \\
Rec. speech age (month) & $38.8 \pm 23.5$ & $50.3 \pm 27.0 *$ & $49.7 \pm 34.2 * * *$ \\
\hline
\end{tabular}

$* \mathrm{P}<0.05$, Baseline compared to 6-week; ** $\mathrm{P}<0.05$, Baseline compared to 12-week;

*** $\mathrm{P}<0.05$, Baseline compared to 6 and 12 -week.

\begin{tabular}{llll}
\hline Group II (Placebo, DH) & $\begin{array}{l}\text { Baseline } \\
(\text { mean } \pm \text { SD) }\end{array}$ & $\begin{array}{l}\text { 6-week } \\
(\text { mean } \pm \text { SD) }\end{array}$ & $\begin{array}{l}\text { 12-week } \\
\text { (mean } \pm \text { SD) }\end{array}$ \\
\hline $\mathrm{n}$ & 20 & 17 & 17 \\
CARS & $35.1 \pm 7.9$ & $32.9 \pm 7.7 *$ & $30.9 \pm 9.1 *$ \\
Exp. speech age (month) & $31.9 \pm 31.1$ & $33.8 \pm 32.9$ & $40.9 \pm 38.8^{* * *}$ \\
Rec. speech age (month) & $33.5 \pm 27.9$ & $39.83 \pm 27$ & $87.37 \pm 14.2 * *$ \\
\hline
\end{tabular}

$* \mathrm{P}<0.05$, Baseline compared to 12-week or Baseline compared to 6-week;

** $\mathrm{P}<0.05,6$-week compared to 12 -week; $* * * \mathrm{P}<0.05$, Baseline compared to both 6 -week and 12 -week.

DH: Donepezil hydrochloride; CARS: Childhood Autism Rating Scale.

\section{Results}

Group I and group II were statistically equivalent at baseline, all $\mathrm{P}<0.05$ (Table 2). Descriptive statistics for group I CARS, EOWPVT-R, and ROWPVT baseline to six-week and twelve-week follow-up are provided in Table 3. Improvements in speech and language were apparent at 6-weeks, and improvements were stable through week 12 when compared to baseline. Improvements in CARS scores are indicated by lowered scores, which were most pronounced by week 12 .

A change of three points or more in the CARS in either direction was considered to be clinically meaningful as it could allow a child to change categorical class, given a baseline average of 34.7.
In group I, $50 \%$ of children had CARS change scores [(Baseline)-(6-week score)] indicating improvement of 3.0 or greater; group II showed that $30 \%$ of CARS scores improved 3 points or greater. Clinically meaningful worsening of CARS scores from baseline to week 6 was apparent in $12.5 \%$ of group I, and $30 \%$ of group II. Item analysis for the CARS did not reveal any specific items within the CARS total score that "carried" the changes in overall scores. With adjustment for multiple comparisons, and to take into consideration the potential for maturation and "learning" with testretest, we adjusted our significance levels with a Tukey's test for multiple comparisons.

Table 3 shows the changes in speech and behavior for children administered placebo for the 
Table 4. Side effects and dropouts

\begin{tabular}{|c|c|c|c|c|}
\hline & $\begin{array}{l}\text { Baseline } \\
\text { DH } \\
\text { Group I }\end{array}$ & $\begin{array}{l}\text { o Week } 6 \\
\text { Placebo } \\
\text { Group II }\end{array}$ & $\begin{array}{l}\text { Week } 6 \text { t } \\
\text { DH } \\
\text { Group I }\end{array}$ & $\begin{array}{l}\text { Week } 12 \\
\text { DH } \\
\text { Group II }\end{array}$ \\
\hline $\begin{array}{l}\text { Diarrhea, stomach cramping } \\
\text { Increased irritability, }\end{array}$ & 0 & 0 & $2(2)$ & 0 \\
\hline mood swings, crying, lability & 5 & 0 & $4(4)$ & 2 \\
\hline More stimming & 0 & 0 & 1 & 0 \\
\hline Lethargy & 1 & 0 & 0 & 0 \\
\hline Frequent urination & 1 & 0 & 0 & 0 \\
\hline Troubled sleep & 1 & 0 & 0 & 0 \\
\hline No-show at 6-week appointment & & & & (3) \\
\hline
\end{tabular}

Numbers of dropouts are indicated in parenthesis.

first six weeks, followed by six weeks of $\mathrm{DH}$.

Table 1 shows the results of combining the six week treatment data for the two groups. The results of the first six weeks from group I were combined with the six weeks of treatment for group II. It is important to note that this combined group therefore contains the data from subjects who were both blinded (group I) and unblinded (group II) to control for any expectancy effects. Statistically significant changes were observed across all three domains. The power and minimum detectable difference were as follows: CARS $(82 \%, 1.70)$, EOWPVT-R $(52 \%$, $4.18)$, and ROWPVT $(87 \%, 6.03)$.

\section{Side Effects}

Table 4 shows side effects reported by the subjects in both groups. Nine patients dropped from the study after the first 6-weeks: 6/9 of these had been on DH when the blinding code was broken. These patients on DH discontinued due to reports of diarrhea or stomach cramping in 2 patients. Increased irritability, accompanied by increased screaming and vocalizations were the most common behavioral complaints in the other 4 patients. Three patients on placebo dropped out due to failure to show up for their 6-week appointment. None of these patients were therefore included in the outcome results. The mean baseline and 6-week results for these patients $(n=9)$ who dropped out are as follows: Baseline CARS (38.4, SD=5.0), EOWPVT-R (47.0, $\mathrm{SD}=17.0)$, and ROWPVT (49.3, $\mathrm{SD}=20.3)$, and 6-week CARS (38.0, SD=6.9), EOWPVT-R (52.0, $\mathrm{SD}=13.8)$, and ROWPVT $(51.7, \mathrm{SD}=27.4)$.

\section{Discussion}

The results of this trial of $\mathrm{DH}$ in pediatric autistic spectrum patients suggest that pharmacological stimulation of acetylcholinergic receptors can improve receptive and expressive language as well as overall general autistic features. Although we cannot conclude that the results were due solely to the addition of $\mathrm{DH}$, several factors lend support to our conclusion that its effect was legitimate. All children had their medications held constant, and no changes to the childrens' environments were undertaken during the study duration (e.g., no new behavioral interventions). Second, the effect of time alone indicates that children with ASD do not follow a typical course of language maturation, since improvement in speech and language, as well as severity of autistic behaviors, did not change significantly for children on placebo. We also conducted a Pearson correlation to evaluate whether age of the subject could have influenced the rate of improvement in expressive or receptive speech but this was not significant $(\mathrm{P}<0.19$ and $\mathrm{P}<$ 0.18 , respectively). Therefore, age maturation alone cannot explain the observed improvements in our test population.

After six weeks on an acetylcholinesterase inhibitor, changes in expressive and receptive speech were: 1. apparent by parental report, 2 . measurable via standardized rating instruments, and 3. statistically significant. There were also parental reports of increased spontaneous non-prompted behaviors that accompanied increased vocalizations and verbalizations, consistent with hypothetical arousal of the frontal cortex and cholinergic innervations. The statistical significance of changes in our measurements of vocabulary and autistic behavior were further supported by parental reports of verbal expressions that appeared to become more laden with emotional content, increased fluency and reduced dysarthria, were also observed when compared to the often non-rhythmic, monotone speech prior to treatment. Greater ease in nonverbal imitation was also frequently reported.

However, there are several important points to note with the data that highlight limitations of the study design. One is that the scores of group I subjects did not continue to show statistically significant increases from 6-weeks to 12 -weeks with DH. The cause of this is not clear, and may need to 
be examined with future long term studies. Also, the receptive speech age of group II increased more than doubled from week 6 to week 12. This dramatic increase was due in part to one subject that was not testable prior to donepezil therapy and scored a 0 for receptive language at baseline and while taking placebo.

Although the use of $\mathrm{DH}$ in pediatric autistic or pervasive developmental disorder patients has not previously been described in a double-blind study, the side effects observed were limited to those reported in prior studies of adult populations (1416). The side effect profile of $\mathrm{DH}$ use in children suggests that in most children it can be tolerated with irritability, hyperactivity, and mild gastrointestinal upset seen in a subset of participants. Decreasing the daily dosage from 2.5 to 1.25 milligrams often ameliorated persistent side effects. The most common adverse event was the concomitant increase in mood swings and lability. Reports of increases in lability, crying, and irritability were often accompanied by reports of increased exploration of environment and increased affection, for example. In many cases, parents viewed these negative temperaments as an "awakening" of the previously aloof affect of their child. Clinically meaningful reports of improvements in expressive and receptive speech can be adequately tracked and measured in this population. Clinical observations supported alterations in exploratory behaviors consistent with those we hypothesized would be affected by $\mathrm{DH}$.

Further long-term use of cholinesterase inhibitors may lead to greater understanding of the possible ability to sustain new neuronal connections in a brain that has more neuronal plasticity than the elderly with Alzheimer's disease.

Continuing study should focus on the behavioral features of ASD that were reported by parents via a more systematic assessment (such as the Autism Diagnostic Observation Scale) to quantify change. The quest for a reliable, standardized, normalized rating instrument to assess clinical efficacy in clinical drug trials in the field of autism remains a current subject of inquiry (17). Small sample size precluded assessment of subgroups of children on varying medication combinations, or analysis of EEG correlates. Further research will help to determine if certain children appreciated more language gains in relationship to drug regimens (i.e., drug-drug interactions). Despite these caveats, DH was observed to improve speech above "placebo effect" reported in other ASD drug trials (18) in both receptive and expressive domains while still having a benign safety profile in this pediatric sample.

\section{References}

1. Perry EK, Lee ML, Martin-Ruiz CM, et al. Cholinergic activity in autism: abnormalities in the cerebral cortex and basal forebrain. Am J Psychiatry 2001; 158: 1058-1066.

2. Bryson HM, Benfield P. Donepezil. Drugs Aging 1997; 10: 234-239.

3. Hoopes S. Donepezil for Tourette's disorder and ADHD. J Clin Psychopharmacol 1999; 19: 381-382.

4. Riback PS. Donepezil for tics in Tourette syndrome. Ann Neurol 2001; 50: S117 (abstract).

5. Kishnani PS, Sullivan JA, Walter BK, et al. Cholinergic therapy for Down's syndrome. Lancet 1999; 353: 1064-1065.

6. Chez MG, Nowinski CV, Buchanan CP. Donepezil use in children with autistic spectrum disorders. Ann Neurol 2000; 48: 541.

7. Hardan AY, Handen HL. A retrospective open trial of adjunctive donepezil in children and adolexcents with autistic disorder. J Child Adolesc Psychopharmacol 2002; 12: 237-241.

8. American Psychiatric Association. Diagnostic and Statistical Manual of Mental Disorders (4th ed). Washington, DC: American Psychiatric Publishing, Incorporated, 1994.

9. Hollander E, Dolgoff-Kaspar R, Cartwright C, Rawitt R, Novotny S. An open trial of divalproex sodium in autism spectrum disorders. J Clin Psychiatry 2001; 62: 530-534.

10. Di Martino A, Tuchman RF. Antiepileptic drugs: affective use in autistic spectrum disorders. Pediatr Neurol 2001; 25: 199-207.

11. Gardner MF. Receptive One-Word Picture Vocabulary Test: ROWPVT. Novato, CA: Academic Therapy Publications, 1985.

12. Gardner MF. Expressive One-Word Picture Vocabulary Test (Revised): EOWPVT-R. Novato, CA: Academic Therapy Publications, 1990.

13. Schopler E, Reichler RJ, Renner BR. The Childhood Autism Rating Scale: CARS. Los Angeles, CA: Western Psychological Sciences, 1988.

14. Greene YM, Noviasky J, Tariot PN. Donepezil overdose. J Clin Psychiatry 1999; 60: 56-57.

15. Rogers SL, Friedhoff LT. The efficacy and safety of donepezil in patients with Alzheimer's disease: results of a US multicenter, Randomized, DoubleBlind, Placebo-Controlled Trial. The Donepezil Study Group. Dementia 1996; 7: 293-303.

16. Wengel SP, Roccaforte WH, Burke WJ, et al. Behavioral complications associated with donepezil. Am J Psychiatry 1998; 155: 1632-1633.

17. Roseman B, Schneider E, Crimmins D, et al. What to measure in autism drug trials. J Autism Dev Disord 2001; 31: 361-362.

18. King BH, Wright DM, Handen BL, et al. Doubleblind, placebo-controlled study of amantadine hydrochloride in the treatment of children with autistic disorder. J Am Acad Child Adolesc Psychiatry 2001; 40: 658-665. 